\title{
Performance of the Rowland Universal Dementia Assessment Scale in Screening Mild Cognitive Impairment at an Outpatient Setting
}

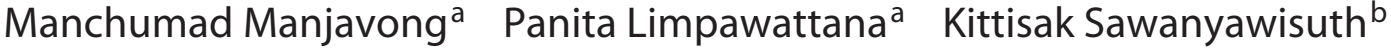 \\ aDivision of Geriatric Medicine, Department of Internal Medicine, Faculty of Medicine, Khon Kaen University, Khon \\ Kaen, Thailand; ${ }^{b}$ Division of Ambulatory Medicine, Department of Internal Medicine, Faculty of Medicine, Khon \\ Kaen University, Khon Kaen, Thailand
}

\section{Keywords}

Aging and cognition · Cognitive assessment · Cognitive disorders · Mild cognitive disorder

\begin{abstract}
Introduction: Mild cognitive impairment ( $\mathrm{MCl})$ is defined as the symptomatic pre-dementia phase on the continuum of cognitive decline. Early recognition and application of potential interventions could prevent or delay the progression to dementia. The Rowland Universal Dementia Assessment Scale (RUDAS) shows good performance in the screening of dementia but has limited data regarding its diagnostic properties in the screening of $\mathrm{MCl}$. The objectives of this study were to assess the psychometric properties of the Thai version of the RUDAS (RUDAS-Thai) in the screening of $\mathrm{MCl}$, identify associated factors for the RUDAS performance, and determine the optimal cutoff point in detecting $\mathrm{MCl}$. Methods: This was a cross-sectional study conducted from January 2020 to March 2021. Older patients at the outpatient clinic of an internal medicine department at a tertiary care hospital in Thailand were examined. Baseline data were collected, and the RUDAS-Thai was administered to each patient. Afterward, a geriatrician assessed each patient for $\mathrm{MCl}$. Results: A total of 150 patients were included, of whom 42
\end{abstract}

karger@karger.com www.karger.com/dee

Karger $\stackrel{\text { ' }}{5}$
(C) 2021 The Author(s)

Published by S. Karger AG, Basel

This is an Open Access article licensed under the Creative Common Attribution-NonCommercial-4.0 International License (CC BY-NC) (http://www.karger.com/Services/OpenAccessLicense), applicable to the online version of the article only. Usage and distribution for commercial purposes requires written permission. cases (28\%) had MCl. The overall performance of the test using an area under the receiver operating characteristic curve (AUC) was 0.82 (95\% confidence interval 0.75-0.89). At the optimal cutoff point of $25 / 30$, the AUC was 0.76 with sensitivity and specificity of 76.2 and $75 \%$, respectively. The educational level affected the test performance according to regression analysis. For patients with years of education $\leq 6$ and $>6$, the optimal cutoff points were $25 / 30$ and $26 / 30$, respectively. Conclusion: The RUDAS-Thai performed well in differentiating patients with $\mathrm{MCl}$ from normal cognition; however, it was affected by educational level. A score of $25 / 30$ or lower for persons with $\leq 6$ years of education or $26 / 30$ or lower for persons with higher than 6 years of education is the optimal cutoff point for indication of developing $\mathrm{MCl}$.

(c) 2021 The Author(s)

Published by S. Karger AG, Basel

\section{Introduction}

Mild cognitive impairment (MCI) is a transitional phase between normal cognitive aging and dementia. It is unlinked to any certain etiology; however, in the context of Alzheimer's disease (AD) biomarkers, it is considered 
to be a prodromal phase of $\mathrm{AD}$. There are several etiologies of MCI such as systemic diseases, neurological diseases, medications, and psychiatric disorders that can cause diverse outcomes [1]. The prevalence of MCI in older adults is around 6.7-25.2\%. It increases with age, lower educational level, and more predominant in men [2-5]. Since MCI was defined differently in most reports, there are wide ranges of its prevalence. The outcomes of MCI can be mainly classified into 3 groups by pathogenesis; reversion to normal aging, stability, or progression to dementia $[1,6-8]$. The annual rate of progression to dementia is approximately $5-17 \%[1,3,8,9]$. Risk factors for predicting progression from $\mathrm{MCI}$ to $\mathrm{AD}$ include a positive amyloid PET scan, apolipoprotein E4 genotype, abnormal CSF tau levels, and a positive PET scan due to tau deposition into the lateral temporal lobe structures [1, $4,9-11]$. Once dementia is diagnosed, it is usually incurable and associated with unfavorable health outcomes as a result of cognitive and functional decline and can complicate other health conditions. Additionally, it can lead to significant health-care costs, socioeconomic, and disability burden, especially in severe cases [12]. Currently, there are only 2 strategies to deal with dementia as follows: symptomatic relief and behavioral intervention [13].

The diagnosis of MCI is primarily on clinical judgment. The 2 main targets are for differentiating MCI from normal aging or dementia and to identify potentially treatable causes of MCI such as depression, medication effects, thyroid disorder, and B12/folate deficiency [5, 14]. In the area of cognitive function assessment, the Montreal Cognitive Assessment (MoCA) is the recommended screening tool for MCI. At a cutoff point of $24 / 30$, its sensitivity and specificity were 80.48 and $81.19 \%$ [15], whereas at the cutoff point of $25 / 30$ had sensitivity of $80-100 \%$ and specificity of $50-76 \%$ [5]. The Rowland Universal Dementia Assessment Scale (RUDAS) is a cognitive screening tool that measures 6 cognitive domains as follows: memory, visuospatial orientation, praxis, visuoconstructional drawing, judgment, and language [16]. It exhibits a good validity, test-retest reliability, and interrater reliability [16-21]. It has been translated in many languages and is not influenced by sex and language, but the educational level affects the test performance in some studies $[17,18]$. The RUDAS score variation with educational attainment; however, it was significantly lesser than MoCA score variation $(p<0.01)$ [22]. The majority of the existing reports study the diagnostic properties in screening dementia, but there is a paucity of data regarding its performance in distinguishing MCI from normal cognitive function [16-21]. The Chinese version of the RUDAS exhibited test-retest reliability of 0.90 with an internal consistency reliability of 0.71 , inter-rater reliability (kappa value) of 0.88 , and a content validity index of 0.97 . The area under the receiver operating characteristic (ROC) curve (AUC) for the MCI assessment was 0.92, and the optimal cutoff point of 24/25 gave sensitivity and specificity of 79 and $91 \%$, respectively. The positive and negative predictive value was 0.99 and 0.96 [20]. Other studies in patients with low- and middle-level education in Peru also showed good diagnostic properties in discrimination patients with MCI from normal cognition and dementia. For the low-educated older adults, the AUC of the RUDAS was $98 \%$ at the optimal cutoff point $<23$ (sensitivity 89 and specificity 93\%) in differentiation MCI from dementia, and it was $98 \%$ at the optimal cutoff point $<19$ (sensitivity 95 and specificity $97 \%$ ) in differentiation dementia from MCI [23]. The AUC of the RUDAS among the middle-education older adults in discrimination patients with MCI from normal cognition was 0.99 at the optimal cutoff point of $<24$ (sensitivity 96 and specificity $90.24 \%$ ), whereas the AUC to discriminate patients with MCI from dementia was 0.89 at the optimal cutoff point of $<21$ (sensitivity 90.24 and specificity $73.81 \%$ ) [19].

In Thailand, the MoCA had been the most commonly used test in detecting MCI though it is influenced by level of education, lifestyle factors, and ethnic diversities [24, $25]$. It also consumes time to administer in real practice. It might not be suitable for bedside and outpatient settings in Thailand. The RUDAS demonstrates good diagnostic performance in MCI detection. As it is shorter than the MoCA, it might be used as an alternate tool. However, its performance in the Thai context has not been addressed. Since early detection of cognitive impairment by a suitable screening tool is crucial and beneficial to global aspects, the study regarding the diagnostic performance of the RUDAS should be performed to lessen the gap of knowledge, identification, and application of potential interventions to prevent or delay the onset of dementia can be established. Additionally, the proceeding of MCI to dementia might be lessened and minimized adverse outcomes of dementia [26]. Therefore, the primary objective of this study was to assess the performance of the RUDAS in discriminating between patients with normal cognitive function and MCI. The secondary objectives were to demonstrate the factors associated with the RUDAS and determine its optimal cutoff point in detecting MCI. 


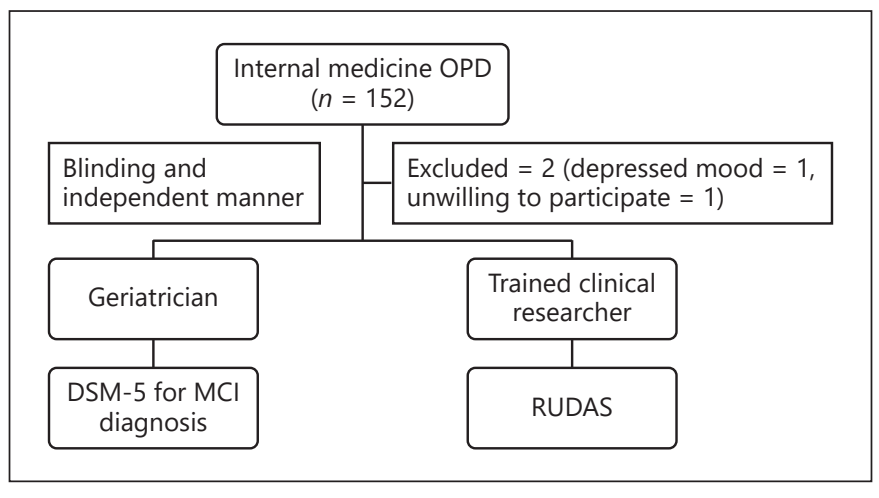

Fig. 1. Study flow. DSM-5, Diagnostic and Statistical Manual of Mental Disorders; MCI, mild cognitive impairment; RUDAS, Rowland Universal Dementia Assessment Scale.

\section{Methods}

\section{Population and Setting}

This is a diagnostic cross-sectional study which was a sub-study of the project entitled "The performance of the RUDAS, Recall test and Mini-Cog in the screening of mild cognitive impairment." The data were collected from older patients who visited the outpatient clinic, Department of Internal Medicine of the Srinagarind University Hospital, Khon Kaen University, Thailand. The study period was between January 2020 and March 2021. The clinic is a tertiary care referral center located in the Northeastern part of Thailand to which patients are sent from other departments and other community hospitals. The inclusion criteria were older adults with age $\geq 60$ years by Thai definition who had no apparent acute illness that can contribute to the performance of the RUDAS such as infection, acute stroke, acute coronary syndrome, and delirium. The exclusion criteria were patients with a psychiatric history, congenital mental retardation, or mental diseases; patients with long-term use of antipsychotic drugs; and patients with severe visual, hearing, or limb dysfunction. Patients with depressed mood determined by the Thai version of the Patient Health Questionnaire-9 $>9$ [27], patients with impaired instrumental activities of daily living determined by the Chula ADL index $<9$ [28], patients who were unable to communicate with Thai or the local language, and the ones who were reluctant to complete the tests were also excluded. The eligible patients were 152 cases where 2 cases were withdrawn due to unwillingness to participate and had one had depressed mood, respectively. The study flow is shown in Figure 1 .

\section{Operational Definition}

Mild Cognitive Impairment

The diagnosis of MCI is based on the definition of the Diagnostic and Statistical Manual of Mental Disorders criteria which were operationalized as follows: (a) evidence of modest cognitive decline from a previous level of performance in one or more cognitive domains which are learning and memory, complex attention, executive function, language, perceptual-motor function, and social cognition, (b) the cognitive deficits do not interfere with the capacity for independence in everyday activities, (c) the cognitive deficits do not occur exclusively in the context of delirium, and (d) the cognitive deficits are not better explained by another mental disorder [29]. The examples of the neuropsychological battery tools used to define impairment of cognitive domains include Mini-Mental State Examination (MMSE), MoCA for global mental functioning, Rey auditory verbal learning test (immediate and delayed recall), short story, Rey-Osterrieth complex figure (recall) for learning and memory domain, trail making test (parts $a$ and b), visual search, symbol digit modalities test stroop and color-word test for attention/executive function domain, phonemic and semantic verbal fluency for language domain, Rey-Osterrieth complex figure (copy) for perceptual motor function domain, and the face and emotion recognition, Faux Pas, or reading the mind in the eyes tests for social cognition domain $[30,31]$.

\section{Instrument}

Rowland Universal Dementia Assessment Scale

The RUDAS is a 6-item cognitive screening test that was developed and validated by Rowland et al. [16, 32]in Australia. This screening tool measures a variety of cognitive domains which tasks included body orientation, praxis (coordination between both hands), copy of cube, a problem-solving task, memory (remembering a shopping list), and semantic verbal fluency (animals). It is easy to administer and can be complete in 10-15 min. The maximum score is 30 , with a higher score reflecting better cognitive performance, and the cutoff points for the Thai version of the RUDAS (RUDAS-Thai) for screening dementia is $23 / 30$ in patients with 6 years of education or lower (sensitivity of 71.4 and specificity of $76.9 \%$ ) and $24 / 30$ in patients with $>6$ years of education (sensitivity of 77 and specificity of $70 \%)[17,33]$.

\section{Procedure}

The potential patients were asked to enroll in the study by a team of researchers. In total, 150 older patients were recruited using convenience sampling. The performance factors including vision, hearing, depression, anxiety, musculoskeletal disorders, fatigue, dysarthria, and dysphasia were reviewed in the medical chart and evaluated by clinical assessment. After consent, demographic information was collected. The RUDAS-Thai version was administered by a trained clinical researcher to the participants where test-retest reliability of the test by the trained clinical researcher was assessed prior to the main study by giving scores to the same patient from the VDO recorder 1 week apart. Then, MCI was assessed by a geriatrician. The trained clinical researcher and the geriatrician were blinded to the results of each other.

\section{Sample Size Calculation}

The required sample size was calculated based on the AUC according to the methodology of Hanley and McNeil [34]. This method altered the sample size until a sufficiently small standard error of the AUC is achieved. A Web-based calculator (www.anaesthetist.com $/ \mathrm{mnm} / \mathrm{stats} / \mathrm{roc} /$ \#stderr) was used to determine the standard error because of the complexity of the formula. Ultimately, a sample size of 150 patients was acceptable and feasible to conduct in clinical practice at the AUC of 0.9 and a standard error of 0.04 . 
Table 1. Demographic data and RUDAS scores of the participants

\begin{tabular}{|c|c|c|}
\hline Variable & $\begin{array}{l}\text { Normal cognitive } \\
\text { function } \\
(n=108)(72 \%)\end{array}$ & $\begin{array}{l}\text { MCI } \\
(n=42)(28 \%)\end{array}$ \\
\hline Age, med (IQR 1, 3) & $67(62.5,73.5)$ & $72(66,75)$ \\
\hline Men, $n(\%)$ & $55(50.9)$ & $17(40.9)$ \\
\hline \multicolumn{3}{|l|}{ Years of education, $n(\%)$} \\
\hline 0 & $0(0)$ & $3(7.1)$ \\
\hline$\leq 6$ & $34(31.5)$ & $25(59.5)$ \\
\hline $6-12$ & $28(25.9)$ & $7(16.7)$ \\
\hline$>12$ & $46(42.6)$ & $7(16.7)$ \\
\hline \multicolumn{3}{|l|}{ Marital status, $n(\%)$} \\
\hline Single & $3(2.8)$ & $2(4.8)$ \\
\hline Married & $82(75.9)$ & $26(61.9)$ \\
\hline Divorce & $8(7.4)$ & $1(2.4)$ \\
\hline Widow & $15(13.9)$ & $13(30.9)$ \\
\hline \multicolumn{3}{|l|}{ Underlying disease(s), $n(\%)$} \\
\hline $\mathrm{DM}$ & $43(39.8)$ & $23(54.8)$ \\
\hline HTN & $81(75)$ & $33(78.6)$ \\
\hline DLD & $72(66.6)$ & $15(35.7)$ \\
\hline CKD & $27(25)$ & $4(9.5)$ \\
\hline $\mathrm{AF}$ & $7(6.5)$ & $4(9.5)$ \\
\hline $\mathrm{IHD}$ & $2(1.9)$ & $2(4.8)$ \\
\hline CVA & $5(4.6)$ & $4(9.5)$ \\
\hline OSA & $7(6.5)$ & $2(4.8)$ \\
\hline RUDAS score, med (IQR 1, 3) & $27.5(25.5,28)$ & $24(22,25)$ \\
\hline
\end{tabular}

$n$, numbers of participants; med, median; IQR, interquartile range; DM, diabetes mellitus; HTN, hypertension; DLD, dyslipidemia; CKD, chronic kidney disease; AF, atrial fibrillation; IHD, ischemic heart disease; CVA, cerebrovascular accident; OSA, obstructive sleep apnea; RUDAS, Rowland Universal Dementia Assessment Scale; MCI, mild cognitive impairment.

\section{Statistical Analysis}

Test-retest reliability of the RUDAS-Thai by a single rater (the trained clinical researcher) was validated by an analysis of limits of agreement, using the Bland and Altman [35] method, by plotting between difference and mean of the RUDAS' scores in a different time. The method of implementation was adjusted until the mean difference from a single rater on 2 separate occasions was not greater than a score of 2 prior proceeding to the main study.

Demographic data variables were summarized using descriptive statistics and were presented as percentage, mean, and standard deviation. If the distribution of these data was not normal, median and interquartile range were used instead. The ROC curve was used to summarize the overall accuracy of RUDAS-Thai for detecting MCI. Then an optimal cutoff point was determined. The performance of the test was summarized by the sensitivity, specificity, positive predictive value, negative predictive value, and likelihood. The effects of age, gender, and education on the RUDASThai were evaluated using linear regression analyses. Variables with $p$ values $<0.05$ were considered to indicate statistically significant coefficients, and $95 \%$ confidence interval were used to determine the strength of association. In addition, an exploratory

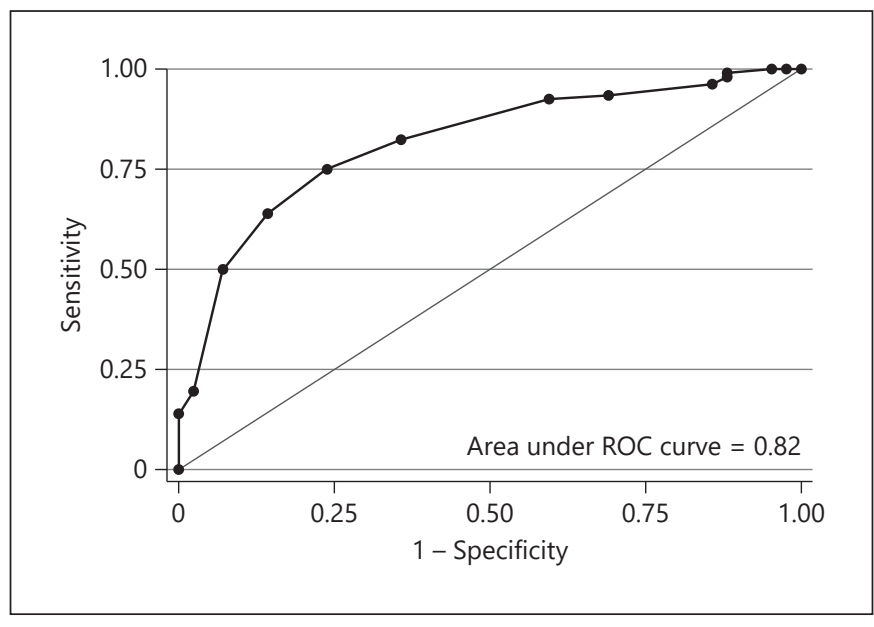

Fig. 2. ROC curve of the RUDAS-Thai for MCI detection. MCI, mild cognitive impairment; AUC, area under the receiver operating characteristic curve; RUDAS, Rowland Universal Dementia Assessment Scale.

data analysis of the RUDAS-Thai performance based on years of education was performed. All of the data analysis will be performed by using STATA version 10.0 (StataCorp, College Station, TX, USA).

\section{Results}

\section{Participant Characteristics}

The demographic data and RUDAS scores of the studied population are demonstrated in Table 1 . The prevalence of patients with MCI was $28 \%$ ( $42 / 150$ cases). The majority of them were women, and they were older and had diabetes mellitus more than the normal cognitive function group. The normal cognitive function group performed higher RUDAS scores than the MCI group.

\section{Performance of the RUDAS-Thai in Detecting Patients with MCI}

The AUC of the RUDAS-Thai was 0.82 (95\% confidence interval 0.75-0.89). The ROC curve is shown in Figure 2. The optimal cutoff point of the test was 25/30, which showed good sensitivity and specificity for MCI detection. The other performances of the test are demonstrated in Table 2.

\section{Effects of Age, Gender, and Educational Level on the \\ RUDAS-Thai}

Only years of education affected the performance of the RUDAS-Thai score on the probability of having MCI, 
Table 2. Optimal cutoff point and its performance of the RUDASThai in detecting of MCI

\begin{tabular}{lcc}
\hline Measure & RUDAS-Thai & $95 \% \mathrm{CI}$ \\
\hline Optimal cutoff point & $\leq 25 / 30$ & \\
At optimal cutoff point & & \\
$\quad$ AUC & 0.76 & $0.68-0.83$ \\
Sensitivity, \% & 76.2 & $60.5-87.9$ \\
Specificity, \% & 75 & $65.7-83.3$ \\
PPV, \% & 54.2 & $40.8-67.3$ \\
NPV, \% & 89 & $80.7-94.6$ \\
Positive LR & 3.1 & $2.1-4.4$ \\
Negative LR & 0.32 & $0.18-0.55$ \\
\hline
\end{tabular}

AUC, area under the receiver operating characteristic curve; $\mathrm{CI}$, confidence interval; PPV, positive predictive value; NPV, negative predictive value; $\mathrm{LR}$, likelihood ratio; $\mathrm{MCI}$, mild cognitive impairment.
Table 3. Factors associated with the performance of the RUDASThai

\begin{tabular}{llll}
\hline Variable & Coefficients & $95 \%$ CI & $p$ value \\
\hline Age, years & -0.05 & $(-0.12$ to 0.03$)$ & 0.23 \\
$\begin{array}{l}\text { Men } \\
\text { Years of education }\end{array}$ & -0.34 & $(-1.32$ to 0.63$)$ & 0.49 \\
$\quad$ & 1 & & \\
$\quad \leq 6$ & 4.6 & $(1.3-7.8)$ & 0.01 \\
$\quad 6-12$ & 5.9 & $(2.6-9.2)$ & 0.001 \\
$>12$ & 6.8 & $(17.1-30.1)$ & 0.00 \\
\hline \multicolumn{2}{c}{ CI, confidence interval. } \\
\hline
\end{tabular}

Table 4. Optimal cutoff points of the RUDAS-Thai for MCI detection based on years of education

\begin{tabular}{lll}
\hline Measure & Six years or lower & Higher than 6 years \\
\hline AUC & $0.74(95 \%$ CI 0.63-0.84) & $\begin{array}{c}0.70(95 \% \text { CI 0.57-0.84) } \\
26 / 30\end{array}$ \\
Optimal cutoff point & $25 / 30$ & \\
At optimal cutoff point & & $71.4 \%(41.9-91.6))$ \\
$\quad$ Sensitivity & $85.7 \%(67.3-96)$ & $68.9 \%(57.1-79.2))$ \\
Specificity & $61.8 \%(43.6-77.8)$ & $30.3 \%(15.6-48.7)$ \\
PPV & $64.9 \%(47.5-79.8)$ & $92.7 \%(82.4-98)$ \\
NPV & $84 \%(63.9-95.5)$ & $2.3(1.42-3.69)$ \\
Positive LR & $2.24(1.42-3.53)$ & $0.42(0.18-0.96)$ \\
Negative LR & $0.23(0.09-0.60)$ & \\
\hline
\end{tabular}

AUC, area under the receiver operating characteristic curve; $\mathrm{CI}$, confidence interval; PPV, positive predictive value; NPV, negative predictive value; LR, likelihood ratio; MCI, mild cognitive impairment. according to the linear regression analysis (Table 3). The performance of the score based on years of education was then analyzed; the AUC of the group with 6 years or lower of education was 0.74 , whereas the other was 0.7 . The optimal cut points were $25 / 30$ and $26 / 30$, respectively. The other performances of the test were shown in Table 4.

\section{Discussion}

The prevalence of MCI in this study was about a third (28\%) which appears to be higher than general reports that found approximately $6.7-25.2 \%$ [2-5]. This difference could be explained by the differences of the studied population. This study was conducted in an outpatient setting of the Internal Medicine department of a tertiary hospital where patients are likely to have more complicated diseases particularly high prevalence of atherosclerotic risk such as diabetes mellitus, hypertension, and dyslipidemia which are the known risk factors for MCI [2]. However, one study regarding the prevalence of MCI in rural Thai older people in the northern part of Thailand reported a very high prevalence of MCI (71.4\%) [36]; the explanation might be due to the criteria for MCI diagnosis in this report (using MoCA-B, Thai version) and characteristics of studied patients (low education and having an underlying disease associated with MCI). Those factors could contribute to the high proportion of MCI in that study.

The overall performance of the RUDAS-Thai in MCI diagnosis in this study was very good based on the AUC (0.82), and the optimal cutoff point was $25 / 30$. The result 
supports the usefulness of the RUDAS, not only for dementia screening but also for MCI detection. Previous studies reported that the early symptoms of MCI and dementia can be effectively identified by assessing a person's visuospatial function which is one of the cognitive domains measured in the RUDAS [16, 32], whereas the MMSE does not measure this domain and the presence of ceiling and floor effects causes modest sensitivity in detecting cognitive decline [37]. However, the educational level, but not age or sex, did affect the performance of the test according to regression analysis. These findings supported the previous report that education influenced the diagnostic properties of the RUDAS-Thai [17]. One explanation could be including problems with visuospatial and visuoconstruction. In comparison to highly educated persons, illiterate persons performed poorer in those cognitive domains which were measured in the RUDAS because of spatial disorganization and lack of 3-dimensionality in this group [38]. The optimal cutoff points were $25 / 30$ and $26 / 30$ for subjects with 6 years or lower education and higher than 6 years of education, respectively. Overall, our finding exhibited rather lower psychometric properties than the previous studies. One study conducted at outpatient clinics of neurology and rehabilitation medicine of the Instituto Peruano de Neurociencias in Lima, Peru, showed the AUC of 0.99 at the optimal cutoff point $<24$, sensitivity 96 , and specificity $90.24 \%$ [19]. Another study which took place at outpatient clinics of the neurology, geriatric, and geriatric psychiatry departments in Taiwan reported the AUC of 0.92 and recommended the cutoff point at 24/30 (AUC 0.85 , sensitivity 79, and specificity 91\%) [39]. These differences may be due to variation in cultural background and language of preference as a prior report regarding the RUDAS-Thai in detecting of dementia performed rather less diagnostic properties than other countries including English and non-English speaking background [39]. For example, some grocery item is not frequently consumed in Southeast Asia [17].

This study revealed that the RUDAS-Thai has satisfactory diagnostic properties in MCI assessment though education did affect its performance where the original one did not $[16,32]$. The RUDAS-Thai then could be an alternate cognitive screening test for MCI in Thai, apart from the MoCA. Further study regarding a direct comparison between the MoCA and the RUDAS is worthwhile. Our results showed that the RUDAS-Thai is an accurate tool for detecting MCI with different cutoff points by education level. Given the cutoff points of the RUDASThai in dementia assessment in the former study [17] were 23 and 24 for subjects with a 6-year or lower education and over a 6-year education, respectively [17], at applying a RUDAS-Thai score of 24-25 for subjects who have 6-year or lower education, and 25-26 for subjects who have over 6-year education are recommended as optimal cutoff points for detection of MCI.

There were some limitations of this study. First, diagnosis bias could be found as the diagnostic criteria for MCI are mainly on a clinical judgment without the accessibility of an easily available biomarker. Second, the prevalence of MCI is likely to have more than expected in general population since our setting is a tertiary care hospital. Subjects are likely to have more complicated illnesses that are at risk for having MCI. Last, as the nature of the study design lacks a longitudinal follow-up and brain pathology, misclassification bias could have occurred.

\section{Conclusion}

The RUDAS-Thai exhibited good diagnostic performance in identifying MCI patients. It was affected by educational level but not by age and sex. The optimal cutoff points at 25/30 for persons with 6 years or lower education and 26/30 for persons with higher than 6 years of education are recommended. The RUDAS-Thai could be an alternative screening test for MCI in an outpatient setting.

\section{Acknowledgments}

We would like to thank the Dementia and its risk factors Research Program, Khon Kaen University, Khon Kaen, Thailand.

\section{Statement of Ethics}

Ethical approval was provided by the Khon Kaen University Faculty of Medicine Ethics Committee as instituted by the Helsinki Declaration (reference number HE621529). All patients have given written informed consent.

\section{Conflict of Interest Statement}

The authors have no conflicts of interest to declare.

\section{Funding Sources}

There was no funding. 


\section{Author Contributions}

P.L. contributed to study design, acquisition of data, analysis and interpretation of data, drafting of the manuscript, and revision of the manuscript for intellectual content. M.M. contributed to study design, acquisition of data, and drafting of the manuscript. K.S. contributed to analysis and interpretation of data, drafting of the manuscript, and revision of the manuscript for intellectual content.

\section{Availability of Data and Material}

All data generated or analyzed during this study are included in its supplementary material file, available at www.karger.com/ doi/10.1159/000517821. Further inquiries can be directed to the corresponding author.

\section{References}

1 Petersen RC, Smith GE, Waring SC, Ivnik RJ, Tangalos EG, Kokmen E. Mild cognitive impairment: clinical characterization and outcome. Arch Neurol. 1999 Mar;56(3):303-8.

2 Jia L, Du Y, Chu L, Zhang Z, Li F, Lyu D, et al. Prevalence, risk factors, and management of dementia and mild cognitive impairment in adults aged 60 years or older in China: a crosssectional study. Lancet Public Health. 2020 Dec;5(12):e661-71.

3 Petersen RC. Clinical practice. Mild cognitive impairment. N Engl J Med. 2011 Jun;364(23): 2227-34.

4 Cheng YW, Chen TF, Chiu MJ. From mild cognitive impairment to subjective cognitive decline: conceptual and methodological evolution. Neuropsychiatr Dis Treat. 2017;13: 491-8.

5 Langa KM, Levine DA. The diagnosis and management of mild cognitive impairment: a clinical review. JAMA. 2014 Dec;312(23): 2551-61.

6 Koepsell TD, Monsell SE. Reversion from mild cognitive impairment to normal or nearnormal cognition: risk factors and prognosis. Neurology. 2012 Oct;79(15):1591-8.

7 Sachdev PS, Lipnicki DM, Crawford J, Reppermund S, Kochan NA, Trollor JN, et al. Factors predicting reversion from mild cognitive impairment to normal cognitive functioning: a population-based study. PLoS One. 2013; 8(3): 559649.

8 Ganguli M, Fu B, Snitz BE, Hughes TF, Chang CC. Mild cognitive impairment: incidence and vascular risk factors in a populationbased cohort. Neurology. 2013 Jun;80(23): 2112-20.

9 Li J-Q, Tan L, Wang H-F, Tan M-S, Tan L, Xu $\mathrm{W}$, et al. Risk factors for predicting progression from mild cognitive impairment to $\mathrm{Alz}$ heimer's disease: a systematic review and meta-analysis of cohort studies. J Neurol Neurosurg Psychiatry. 2016 May;87(5):476-84.

10 Yarnall AJ, Rochester L, Burn DJ. Mild cognitive impairment in Parkinson's disease. Age Ageing. 2013 Sep;42(5):567-76.

11 Farias ST, Mungas D, Reed BR, Harvey D, DeCarli C. Progression of mild cognitive impairment to dementia in clinic- vs communitybased cohorts. Arch Neurol. 2009 Sep;66(9): 1151-7.
12 Witthaus E, Ott A, Barendregt JJ, Breteler M, Bonneux L. Burden of mortality and morbidity from dementia. Alzheimer Dis Assoc Disord. 1999 Jul-Sep;13(3):176-81.

13 Karakaya T, Fußer F, Schröder J, Pantel J. Pharmacological treatment of mild cognitive impairment as a prodromal syndrome of Alzheimer's disease. Curr Neuropharmacol. 2013 Jan;11(1):102-8.

14 Jongsiriyanyong S, Limpawattana P. Mild cognitive impairment in clinical practice: a review article. Am J Alzheimers Dis Other Demen. 2018;33(8):500-7.

15 Espino DV, Lichtenstein MJ, Palmer RF, Hazuda HP. Ethnic differences in mini-mental state examination (MMSE) scores: where you live makes a difference. J Am Geriatr Soc. 2001 May;49(5):538-48.

16 Storey JE, Rowland JT, Basic D, Conforti DA, Dickson HG. The rowland universal dementia assessment scale (RUDAS): a multicultural cognitive assessment scale. Int Psychogeriatr. 2004 Mar; 16(1):13-31.

17 Limpawattana P, Tiamkao S, Sawanyawisuth $\mathrm{K}$. The performance of the rowland universal dementia assessment scale (RUDAS) for cognitive screening in a geriatric outpatient setting. Aging Clin Exp Res. 2012 Oct;24(5): 495-500.

18 Iype T, Ajitha BK, Antony P, Ajeeth NB, Job $S$, Shaji KS. Usefulness of the rowland universal dementia assessment scale in South India. J Neurol Neurosurg Psychiatry. 2006 Apr; 77(4):513-4.

19 Custodio N, Montesinos R, Lira D, HerreraPerez E, Chavez K, Hernandez-Córdova G, et al. Validation of the RUDAS in patients with a middle-level education in Lima, Peru. Am J Alzheimers Dis Other Demen. 2019 NovDec;34(7-8):513-22.

20 Chu LW, Ng KH, Law AC, Lee AM, Kwan F. Validity of the cantonese Chinese montreal cognitive assessment in Southern Chinese. Geriatr Gerontol Int. 2015 Jan;15(1):96-103.

21 Goudsmit M, van Campen J, Schilt T, Hinnen C, Franzen S, Schmand B. One size does not fit all: comparative diagnostic accuracy of the rowland universal dementia assessment scale and the mini mental state examination in a memory clinic population with very low education. Dement Geriatr Cogn Dis Extra. 2018 May-Aug;8(2):290-305.
22 Brymer C, Sider C, Evans A, Lee BY, Taneja $\mathrm{K}$, Morgenstern J, et al. Montreal cognitive assessment vs. rowlnad dementia assessment scale for cognitive screening. Innov Aging. 2017 Jul;1(Suppl 1):468.

23 Custodio N, Montesinos R, Lira D, HerreraPerez E, Chavez K, Reynoso-Guzman W, et al. Validation of the RUDAS for the identification of dementia in illiterate and low-educated older adults in Lima, Peru. Front Neurol. 2020 May;11:374.

24 Gagnon G, Hansen KT, Woolmore-Goodwin S, Gutmanis I, Wells J, Borrie M, et al. Correcting the MoCA for education: effect on sensitivity. Can J Neurol Sci. 2013 Sep;40(5): 678-83.

25 O'Driscoll C, Shaikh M. Cross-cultural applicability of the montreal cognitive assessment (MoCA): a systematic review. J Alzheimers Dis. 2017;58(3):789-801.

26 Lowry KA, Vallejo AN, Studenski SA. Successful aging as a continuum of functional independence: lessons from physical disability models of aging. Aging Dis. 2012 Feb;3(1): 5-15.

27 Lotrakul M, Sumrithe S, Saipanish R. Reliability and validity of the Thai version of the PHQ-9. BMC Psychiatry. 2008 Jun;8:46.

28 Jitapunkul S. Disability: the elderly problems. Chula Med J. 1994;38(2):68-75.

29 Sachdev PS, Blacker D, Blazer DG, Ganguli $\mathrm{M}$, Jeste DV, Paulsen JS, et al. Classifying neurocognitive disorders: the DSM-5 approach. Nat Rev Neurol. 2014 Nov; 10(11):634-42.

30 Salvadori E, Poggesi A, Pracucci G, Chiti A, Ciolli L, Cosottini M, et al. Application of the DSM-5 criteria for major neurocognitive disorder to vascular MCI patients. Dement Geriatr Cogn Disord EXTRA. 2018 Mar;8(1):10416.

31 Macías Islas MÁ, Ciampi E. Assessment and impact of cognitive impairment in multiple sclerosis: an overview. Biomedicines. 2019 Mar;7(1):22.

32 Pang J, Yu H, Pearson K, Lynch P, Fong C. Comparison of the MMSE and RUDAS cognitive screening tools in an elderly inpatient population in everyday clinical use. Intern Med J. 2009 Jun;39(6):411-4. 
33 Limpawattana P, Tiamkao S, Sawanyawisuth K, Thinkhamrop B. Can rowland universal dementia assessment scale (RUDAS) replace mini-mental state examination (MMSE) for dementia screening in a Thai geriatric outpatient setting? Am J Alzheimers Dis Other Demen. 2012 Jun;27(4):254-9.

34 Hanley JA, McNeil BJ. A method of comparing the areas under receiver operating characteristic curves derived from the same cases. Radiology. 1983 Sep;148(3):839-43.
35 Bland JM, Altman DG. Statistical methods for assessing agreement between two methods of clinical measurement. Lancet Lond Engl. 1986 Feb;1(8476):307-10.

36 Griffiths J, Thaikruea L, Wongpakaran N, Munkhetvit P. Prevalence of mild cognitive impairment in rural Thai older people, associated risk factors and their cognitive characteristics. Dement Geriatr Cogn Disord Extra. 2020 Apr;10(1):38-45.

37 Franco-Marina F, García-González JJ, Wagner-Echeagaray F, Gallo J, Ugalde O, Sánchez-García S, et al. The mini-mental state examination revisited: ceiling and floor effects after score adjustment for educational level in an aging Mexican population. Int Psychogeriatr. $2010 \mathrm{Feb} ; 22(1): 72-81$.
38 Julayanont P, Ruthirago D. The illiterate brain and the neuropsychological assessment: from the past knowledge to the future new instruments. Appl Neuropsychol Adult. 2018 Mar-Apr;25(2):174-87.

39 Chen CW, Chu H, Tsai CF, Yang HL, Tsai JC, Chung $\mathrm{MH}$, et al. The reliability, validity, sensitivity, specificity and predictive values of the Chinese version of the rowland universal dementia assessment scale. J Clin Nurs. 2015 Nov;24(21-22):3118-28. 